\title{
A fundamental figure of merit for radio polarimeters
}

\author{
T. D. Carozzi and G. Woan
}

Department of Physics and Astronomy, University of Glasgow, Glasgow G12 8QQ

Received 17 Aug 2009

\begin{abstract}
Context. Modern radio astronomy requires high-precision polarimetry and makes heavy demands on the design and construction of radio polarimeters (telescope systems that can measure the polarization state of sources). It would therefore be desirable to have a figure of merit (FoM) with which to assess the polarimetric performance of these instruments. The parameter commonly used for this purpose, the cross-polarization ratio, is not well-suited as a FoM as it depends on the choice of coordinate system used for the polarimetry. In this way a given polarimeter can have almost any cross-polarization ratio.

Aims. To introduce a fundamental FoM for radio polarimeters specific to polarimetry.

Methods. We use the singular values of the measurement matrices of the polarimeter, either Jones matrix or Mueller matrix, to define an invariant cross-polarization parameter.

Results. We find that the polarimeter intrinsic cross-polarization ratio (IXR) can be used as a fundamental FoM for a polarimeter. We give real examples of the IXR for polarimeters such as the Parkes radio telescope, the Westerbork Synthesis radio telescope, the Effelsberg telescope, and dipole array interferometers (simple model for future telescopes such as LOFAR and SKA).

Conclusions. The polarimeter IXR is found to be related to the polarimeters measurement matrix's condition number, and so it is a crucial parameter in the total relative error in a polarimeter's measurements. We find that it can also be used to assess the calibration of a polarimeter and in determining the quality of an interferometric measurement equation.
\end{abstract}

Key words. Instrumentation: polarimeters

\section{Introduction}

Precision polarimetry is now an integral part of modern radio science. Indeed, many of the key scientific objectives for existing and future radio telescopes rely on highly accurate measurements of the full state-of-polarization of celestial signals. This in turn places tough polarimetric requirements on the radio polarimeters and their design. These polarimetric requirements are usually specified in terms of the cross-polarization ratio of the polarimeter, which is often taken as a figure of merit (FoM) of the polarimeter.

Cross-polarization ratio is an IEEE radio standard definition. Based on a dual-polarized antenna model of a polarimeter, the cross-polarization ratio is, loosely speaking, the ratio of the desired co-polarized power to the undesired cross-polarized power. There are many possible reasons for a polarimeter to exhibit cross-polarization including for example non-orthogonal antennas, electronic leakage between channels, and mutual-coupling between antennas. If the IEEE defined parameter actually were a consistent measure of these types of phenomena then it would be a suitable FoM of a polarimeter. However, it is our belief that the standard definition is not suitable as a FoM, for the following reasons: First, the standard cross-polarization ratio varies with the choice of coordinates and so it is not a measure of polarimeter intrinsic (that is, independent of coordinate system) properties. Second, cross-polarization is generally not relevant in a fully calibrated polarimeter since if properly calibrated, virtually all cross-polarization can be removed, at least in principle.

This second point raises a fundamental question posed by, for instance, Thiel (1976): is the performance of a polarimeter ultimately arbitrary, in the sense that systematic errors can be eliminated numerically with a full calibration? Thiel (1976) gives good reasons why the answer to this question should be negative, but no concise parameter for assessing the a polarimeter's performance is provided.

In this paper we introduce a FoM parameter, which we call the polarimeter intrinsic cross-polarization ratio (IXR), that addresses these two points. It is a cross-polarization ratio defined independently of coordinate system with properties that are relevant to polarimeters. It can be understood as the worst-case cross-polarization ratio of a given polarimeter before calibration, and it is closely related to the total relative error of the fully calibrated polarimeter. The IXR is therefore well-suited as a fundamental polarimeter FoM.

\section{Fundamentals of radio polarimetry}

By definition, a polarimeter is an instrument which measures some aspect of the polarization of electromagnetic radiation. However, in what follows we will consider only polarimeters that are capable of measuring the full state of polarization of the radiation, at least for some incidence direction. This can either be stated in terms of the two, complex-valued, transverse components of the electric field comprising the Jones vector, or the four real-valued Stokes parameters. If a polarimeter measures the full Jones vector we will refer to it as a Jones polarimeter, and if the polarimeter measures the full set of Stokes parameters we will refer to it as a Stokes polarimeter. Often a polarimeter can been seen as both a Jones and a Stokes polarimeter, and in these cases we will refer to such a device as a Jones/Stokes polarimeter. Furthermore, we will only consider Jones/Stokes polarimeters with linear responses.

Linear Jones polarimeters are modelled by a simple linear relationship between the true Jones vector at the input of the polarimeter, e, and the measured Jones vector at the output of 
the polarimeter, $\mathbf{f}$, i.e.,

$$
\mathbf{f}(\omega, \mathbf{s})=\mathbf{J}(\omega, \mathbf{s}) \mathbf{e}(\omega, \mathbf{s}) .
$$

Here, $\mathbf{J}$ is the response, or Jones, matrix of the polarimeter, $\omega$ is the angular frequency of the incident radio wave associated with the true Jones vector and $\mathbf{s}$ is the unit vector along the direction of arrival of the radio wave. We take $\mathbf{s}=\mathbf{s}_{0}$ to be the direction of the polarimeters boresight, also known as the on-axis direction. For simplicity we will suppress these $(\omega, \mathbf{s})$ dependencies in the analysis below. If an explicit Jones matrix is considered then it will be assumed to be with reference to the direction $\mathbf{s}=\mathbf{s}_{0}$ and that $\omega$ corresponds to the centre frequency of the polarimeter's receiver.

In the basic polarimeter response relation, Eq. (1), we take the complex components of $\mathbf{e}$ to be with respect to a real Cartesian basis $\{\hat{\mathbf{x}}(\mathbf{s}), \hat{\mathbf{y}}(\mathbf{s})\}$, which depends on $\mathbf{s}$, and we write the components of the Jones vector as

$$
\mathbf{e}(\mathbf{s})=\left(\begin{array}{c}
e_{x}(\mathbf{s}) \\
e_{y}(\mathbf{s})
\end{array}\right), \quad \text { w.r.t. }\{\hat{\mathbf{x}}(\mathbf{s}), \hat{\mathbf{y}}(\mathbf{s})\} .
$$

We will refer to this basis as the local sky basis for $\mathbf{s}$, denoted $\mathcal{S}(\mathbf{s}) \equiv\{\hat{\mathbf{x}}(\mathbf{s}), \hat{\mathbf{y}}(\mathbf{s})\}$, and for the special case $\mathbf{s}=\mathbf{s}_{0}$ this will be denoted $\mathcal{S}_{0}$. The nominal local sky basis, $\mathcal{S}$, is the standard local basis given by the coordinate system used for observations. Examples are the spherical altitude-azimuth coordinate system, or the coordinate systems based on Ludwig's definitions in Ludwig (1973). f, on the left-hand side of Eq. (1), is also a two-dimensional complex vector. Its components are associated with the two polarimeter channels, denoted $p$ and $q$, and we write

$$
\mathbf{f}=\left(\begin{array}{c}
f_{p} \\
f_{q}
\end{array}\right)
$$

with respect to the nominal basis $\{\hat{\mathbf{p}}, \hat{\mathbf{q}}\}$, which we will call the channel basis $C$ and refer to channels $p, q$ respectively. The Jones matrix in Eq. (1) maps vectors with respect to $\mathcal{S}(\mathbf{s})$ to vectors with respect to $C$, so we write its components as

$$
\mathbf{J}=\left(\begin{array}{ll}
\mathrm{J}_{p x} & \mathrm{~J}_{p y} \\
\mathbf{J}_{q x} & \mathrm{~J}_{q y}
\end{array}\right) \text {. }
$$

The Jones matrix components depend on $\mathcal{S}(\mathbf{s})$. If we change the local sky basis to $\mathcal{S}^{\prime}(\mathbf{s})$, then they transform as

$$
\mathbf{f}=\mathbf{J V}^{\dagger} \mathbf{V e}=\mathbf{J}^{\prime} \mathbf{e}^{\prime}
$$

where $\mathbf{V}^{\dagger}$ is the Hermitian transpose of $\mathbf{V} . \mathbf{V}$ is the unitary matrix (i.e., $\mathbf{V}^{\dagger} \mathbf{V}=\mathbf{I}$ where $\mathbf{I}$ is the unit matrix) that specifies the unitary transformation between $\mathcal{S}$ and $\mathcal{S}^{\prime}$. Note that generally $\mathcal{S}^{\prime}$ is not the standard local basis associated with the global coordinate system. Also, since we are considering unitary transformations, $\mathcal{S}^{\prime}$ may not be a set of real Cartesian base vectors, and so we will not denote its components $x$ and $y$, but rather 1 and 2, respectively. Of course this change of basis has no effect at all on the measurements of the components of $\mathbf{f}$, but it does affect their interpretation.

Similarly, the Jones matrix and the measured Jones vector under changes in the channel basis $C$ are transformed as

$$
\mathbf{f}^{\prime}=\mathbf{U f}=\mathbf{U} \mathbf{J e}=\mathbf{J}^{\prime} \mathbf{e},
$$

where $\mathbf{U}$ is another unitary matrix. In contrast to changing the sky basis, changing the channel basis is an active transformation since it requires transforming the output through a matrix multiplication with $\mathbf{U}$. Despite being an active transformation, it does not distort the polarimetry, as it is a unitary transformation. Note that this transformation need not involve the polarimeter at all since it can be performed with high precision on the numerical output.

The most general transformation of Eq. (1) is a simultaneous change in both local sky basis and the channel basis given by

$$
\mathbf{f}^{\prime}=\mathbf{J}^{\prime} \mathbf{e}^{\prime},
$$

where

$$
\begin{aligned}
\mathbf{f}^{\prime} & =\mathbf{U f}, \\
\mathbf{e}^{\prime} & =\mathbf{V e}, \\
\mathbf{J}^{\prime} & =\mathbf{U} \mathbf{J} \mathbf{V}^{\dagger} .
\end{aligned}
$$

This relationship between $\mathbf{J}$ and $\mathbf{J}^{\prime}$ implies that they are unitarily equivalent matrices (Lancaster \& Tismenetsky 1985). If $\mathbf{U}=\mathbf{V}$, then $\mathbf{J}$ and $\mathbf{J}^{\prime}$ are unitarily similar matrices. Therefore, in general, the Jones matrix of a polarimeter can be any unitarily equivalent matrix to the original $\mathbf{J}$, which is given with respect to $\mathcal{S}$ and $C$.

We define an ideal polarimeter to be one that has a Jones matrix that is exactly proportional to a unitary matrix. This is ideal because a unitary matrix does not distort the true Jones vector, it is merely a change of the orthonormal basis.

In reality, a polarimeter is never ideal, and so to obtain the true Jones vector one should invert Eq. (1) to give

$$
\mathbf{e}=\mathbf{J}^{-1} \mathbf{f} .
$$

In this context, the full calibration of a Jones polarimeter is the process of determining the polarimeter's $\mathbf{J}$, inverting it, and matrix-multiplying it with the measured data. The existence of this solution is guaranteed here since we only consider full Jones polarimeters, which by definition will have invertible Jones matrices at least for some direction.

Although the solution given by Eq. (4) is in principle exact, it assumes that $\mathbf{f}$ and $\mathbf{J}$ are known exactly. In reality noise corrupts the polarimeter measurements so that $\mathbf{f}$ and $\mathbf{J}$ are known with only limited precision, and this in turn limits the precision with which we can determine e. There are, however, Jones matrices for which the relative error in the estimated $\mathbf{e}$ is less sensitive to noise than for others. From matrix algebra theory this sensitivity is determined by the condition number of $\mathbf{J}$, defined as

$$
\operatorname{cond}(\mathbf{J})=\|\mathbf{J}\|\left\|\mathbf{J}^{-1}\right\|,
$$

where $\|\mathbf{J}\|$ is some matrix norm (Lancaster \& Tismenetsky 1985). For the spectral norm, or 2 -norm, the condition number $k$ of $\mathbf{J}$ is

$$
\operatorname{cond}_{2}(\mathbf{J}) \equiv \kappa(\mathbf{J})=\frac{\sigma_{\mathrm{M}}}{\sigma_{\mathrm{m}}},
$$

where $\sigma_{\mathrm{M}}$ and $\sigma_{\mathrm{m}}$ are the largest and smallest singular values of $\mathbf{J}$, respectively. Although other norms could be adopted we will only consider the spectral norm since, as we shall see, it is easily related to the polarimeters intrinsic cross-polarization ratio.

There are some important properties of the condition number that we will use. We see from Eq. (5) that $\kappa$ is real and never smaller than 1, i.e.,

$$
1 \leq \kappa(\mathbf{J}) .
$$

Also, the condition of a unitary matrix is equal to the greatest lower bound for the condition number, i.e., for any complex matrix $\mathbf{J}$ and unitary matrix $\mathbf{U}$,

$$
\operatorname{cond}(\mathbf{U}) \leq \operatorname{cond}(\mathbf{J}) .
$$


This gives additional, quantitative, support for our requirement that an ideal polarimeter have a unitary Jones matrix. In terms of the condition number there is no better matrix for the inversion of Eq. (1). A final property we shall use is that the condition of a matrix is not affected by a unitary transformation, i.e.,

$$
\operatorname{cond}(\mathbf{J U})=\operatorname{cond}(\mathbf{J}) \text {. }
$$

The condition number of a polarimeter's Jones matrix can be seen as a FoM for a polarimeter. Although this measure is not traditionally used in radio astronomy it is to be found in optics (Sabatke et al. 2000). In practice, the radio astronomical polarimetry community uses the polarimeter's cross-polarization ratio instead. Let us now introduce this concept.

\section{Existing definitions of cross-polarization and feed leakage}

The concept of cross-polarization is widely used in the analysis of polarimeter performance as is evident from the large number synonymous terms used to refer to it, including feed leakage, polarization purity, $D$-terms, instrumental polarization, cross-coupling, mutual-coupling, channel cross-talk, crosspolarization isolation, cross-polarization discrimination and so on (Reid et al. 2008; Massi et al. 1997; Sault et al. 1996; Leppänen et al. 1997). All these terms are associated with the situation in which a fraction of one polarimetric component is added to the other component, and without compensation this will lead to erroneous polarimetry. This long list of similar terms should convince the reader that there is a need for standardising and consolidating a concept which we will simply call crosspolarization. Having said that, the last two concepts in the list (cross-polarization isolation and cross-polarization discrimination) are part of radio engineering standards (IEEE 1998), and do have clear definitions.

The standard cross-polarization ratio is used as a specification for radio polarimeters, and is often interpreted as a FoM for the polarimeter quantifying the amount of detrimental leakage that is acceptable. Clearly there is a need for a FoM specific to the polarimetric performance of the polarimeter, but is the standard cross-polarization ratio parameter suitable? We now briefly review these standard definitions of cross-polarization from the perspective of radio astronomy. In doing so we will use terminology and formalism of Hamaker et al. (1996).

A Jones matrix model of a polarimeter can be given as follows: labeling the two polarimeter channels $p$ and $q$, the Jones matrix is factored as

$$
\mathbf{J}=\mathbf{G D C}
$$

where

$$
\mathbf{G}=\left(\begin{array}{cc}
g_{p} & 0 \\
0 & g_{q}
\end{array}\right)
$$

is the complex receiver gain,

$$
\mathbf{D}=\left(\begin{array}{cc}
1 & d_{p} \\
-d_{q} & 1
\end{array}\right)
$$

is the feed-error, or leakage, matrix and $\mathbf{C}$ is the idealized nominal feed configuration. We assume that $\mathbf{C}$ is a given unitary matrix so it is known exactly a priori.

In this model, the off-diagonal parameters $d_{p}$ and $d_{q}$ are seen as being related to cross-polarization, or leakage terms (see e.g. Hamaker et al. (1996)). Due to $\mathbf{D}$, the signal at the input of one channel finds its way to the output of the other channel, and vice versa. Specifically, regard channel $p$ as the co-polarized component. The ratio of the power in the co-polarized channel output from a purely co-polarized unit power source to the power in the co-polarized channel output from a purely cross-polarized unit power source is $1 /\left|d_{p}\right|^{2}$. The analogous ratio for channel $q$ gives $1 /\left|d_{q}\right|^{2}$. These parameters are the cross-polarization isolations (XPIs) of channels $p$ and $q$ respectively(Stutzman 1993). Importantly, XPI is an IEEE radio standard (IEEE 1998) and is a recognised way of specifying the cross-polarization of a polarimeter.

The IEEE standard also defines a dual to XPI called crosspolarization discrimination (XPD). XPD can be seen as the alternative factorisation of $\mathbf{J}$

$$
\mathbf{J}=\mathbf{D}^{\prime} \mathbf{G}^{\prime} \mathbf{C}
$$

where

$$
\mathbf{G}^{\prime}=\left(\begin{array}{cc}
g_{1}^{\prime} & 0 \\
0 & g_{2}^{\prime}
\end{array}\right)
$$

and

$$
\mathbf{D}^{\prime}=\left(\begin{array}{cc}
1 & d_{2}^{\prime} \\
-d_{1}^{\prime} & 1
\end{array}\right) \text {. }
$$

The XPDs of $\mathbf{J}$ are the reciprocals of $\left|d_{1}^{\prime}\right|^{2}$ and $\left|d_{2}^{\prime}\right|^{2}$ with respect to components 1 and 2 of the Jones vector with basis vectors given by the configuration matrix $\mathbf{C}$. In general $\mathbf{G}^{\prime}$ and $\mathbf{D}^{\prime}$ will not be equal to $\mathbf{G}$ and $\mathbf{D}$ so the two XPDs will be different from two XPIs.

The model expressed in Eq. (6), or alternatively Eq. (7), is very flexible and can express every possible configuration. Unfortunately it is precisely this flexibility that makes it difficult to discern the extrinsic, coordinate system dependent, properties of the polarimeter from the intrinsic, coordinate system independent, properties. Consider for instance a change in the channel basis, see Eq. (3), in which the sum and the difference of channels $p$ and $q$ are taken as two new channels. It is not difficult to see that such a change, which is in fact sometimes employed in practice, will change all of the XPIs and XPDs. Indeed, take a perfectly orthonormal pair of receptors which in some coordinate system will have an XPI of 0 , and rotate that system through $45^{\circ}$. One finds that this rotated system has an XPI of $100 \%$. So even though nothing has changed electrically or mechanically with the polarimeter, it would seem that its leakage, as quantified by the XPI, has changed from one extreme to the other. This illustrates that XPI and XPD are extrinsic properties of the polarimeter, with a large dependence on the arbitrary choice of the local basis.

\section{Intrinsic cross-polarization ratio $\left(\mathrm{IXR}_{\mathrm{J}}\right)$ of Jones polarimeters}

Having found that XPI and XPD are extrinsic properties of a polarimeter, we now ask whether it is possible to define a intrinsic measure of cross-polarization. By this we mean a parameter analogous to XPI or XPD, but which does not vary with the choice of basis. We do this by finding a matrix unitarily equivalent to the polarimeter's Jones matrix which is invariant.

Say we are given the on-axis Jones matrix, $\mathbf{J}=\mathbf{J}\left(\mathbf{s}_{0}\right)$, with respect to channel basis $C$ and the on-axis basis $\mathcal{S}_{0}$, for some polarimeter. We introduce a unique Jones matrix $\breve{J}$ unitarily similar to $\mathbf{J}$ that can be factored as

$$
\check{\mathbf{J}}=\mathbf{T}^{\dagger} \mathbf{J} \mathbf{T}=G \check{\mathbf{D}} \check{\mathbf{C}}
$$



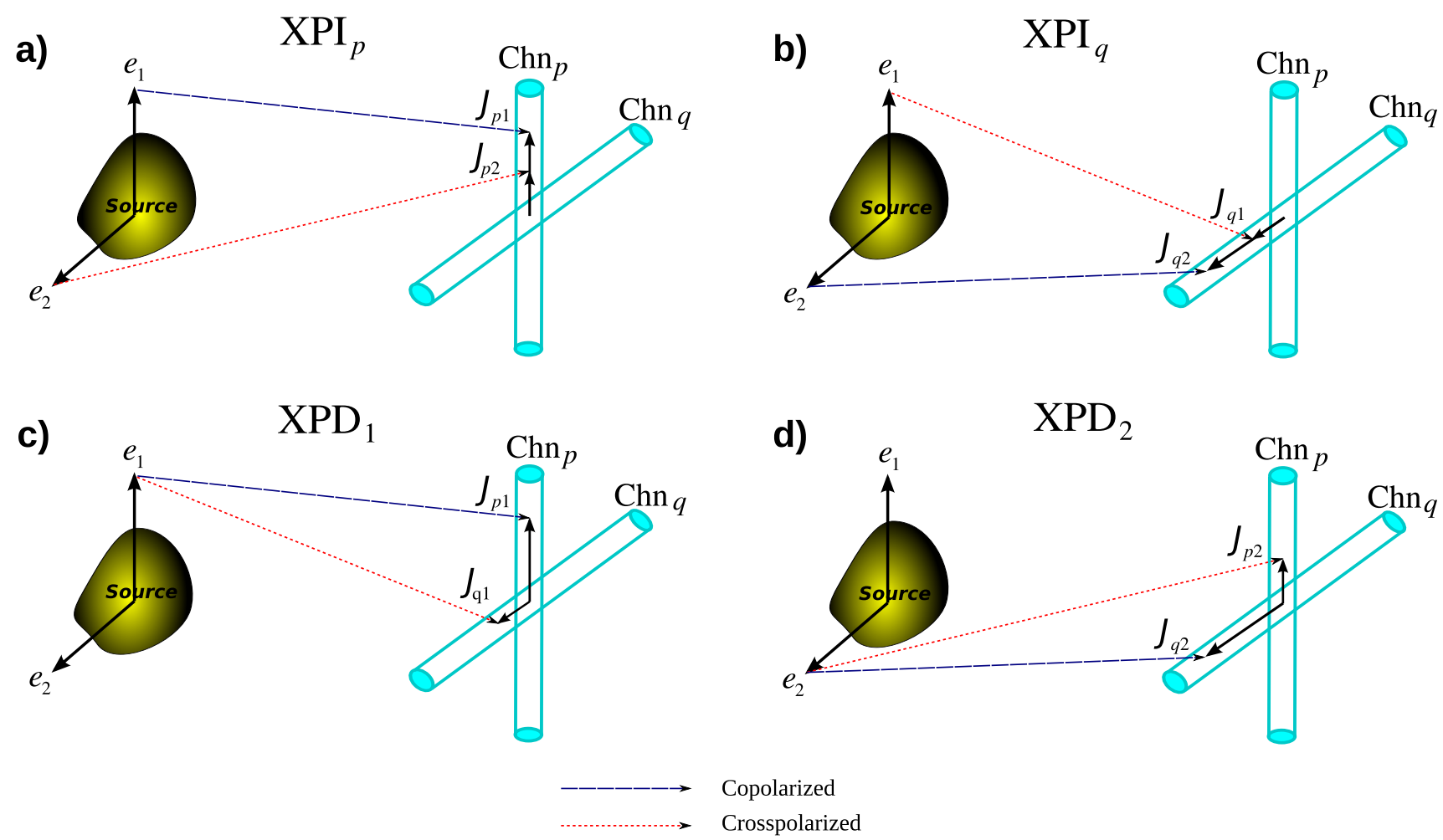

Figure 1. Cross-polarization definitions in a polarimeter. These figurative sketches show the components of the polarimeter's Jones matrix $\mathbf{J}$ for channels $p$ and $q$ due to the components 1 and 2 of the Jones (transverse electric) vector. Nominally, components 1 and 2 correspond to the basis vectors given by the complex conjugate of the first and second rows, respectively, of the polarimeter's feed configuration matrix $\mathbf{C}$. The polarimeter channels are shown symbolically as cylinders labeled "Chn". The arrows represent (symbolically) the complex output signal from these channels due to the corresponding Jones vector components. The cross-polarization isolation (XPI) for $p$ and $q$ are shown in a) and b), respectively computed as $\left|\mathrm{J}_{p 1} / \mathrm{J}_{p 2}\right|^{2}$ and $\left|\mathrm{J}_{q 1} / \mathrm{J}_{q 2}\right|^{2}$. The cross-polarization discrimination (XPD) for components 1 and 2 are shown in c) and d), respectively computed as $\left|J_{p 1} / J_{q 1}\right|^{2}$ and $\left|J_{p 2} / J_{q 2}\right|^{2}$.

where $G$ is a positive real scalar, $\check{\mathbf{C}}$ is a unitary matrix and

$$
\check{\mathbf{D}} \equiv \frac{1}{\sqrt{1+d^{2}}}\left(\begin{array}{ll}
1 & d \\
d & 1
\end{array}\right),
$$

where $0 \leq d \leq 1$. Furthermore,

$$
G \equiv \sqrt{\frac{\sigma_{\mathrm{M}}^{2}+\sigma_{\mathrm{m}}^{2}}{2}}
$$

and

$$
d \equiv \frac{\sigma_{\mathrm{M}}-\sigma_{\mathrm{m}}}{\sigma_{\mathrm{M}}+\sigma_{\mathrm{m}}}
$$

where $\sigma_{\mathrm{M}}$ and $\sigma_{\mathrm{m}}$ are the largest and smallest singular values of $\mathbf{J}$ respectively. One can easily prove that the decomposition in Eq. (8) is always possible using the singular value decomposition theorem (Lancaster \& Tismenetsky 1985) on J. Clearly Eq. (8) is analogous to Eq. (6), the important difference is that decomposition in Eq. (8) is invariant. The unique factorisation of the Jones matrix $\mathbf{J}$ specifies a unique local basis, given by the transformation matrix $\mathbf{T}$, rather than an arbitrary basis such as $C$ and $\mathcal{S}_{0}$. Its manifestly obvious features is that its gain matrix, $\mathbf{G}=G \mathbf{I}$, is a scalar and that its leakage matrix, $\mathbf{D}=\check{\mathbf{D}}$, is symmetric along both diagonals, thus all the XPI and XPD of $\mathbf{J}^{\prime}$ are equal.

Indeed, each of the factors in factorisation above is unique. Let us go them one by one and highlight their relevance to radio polarimetry.
The amplitude gain $G$ in Eq. (8) is exactly the positive square root of the total power gain of the polarimeter for an unpolarized source. This makes $G$ unique.

The feed configuration matrix Č in Eq. (8) is the Jones ma-

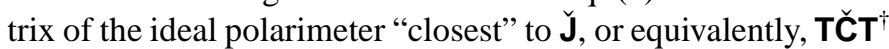
is the unitary matrix closest to $\mathbf{J}$. Closeness in terms of matrix distance is defined as $\|\mathbf{A}-\mathbf{B}\|$ where $\|\cdot\|$ is a unitarily invariant matrix norm (Fan \& Hoffman 1955), and so two matrices are closest to each other when the matrix distance between them is minimal. If $\mathbf{J}$ has the polar decomposition

$$
\mathbf{J}=\overline{\mathbf{J}} \overline{\mathbf{C}}
$$

where $\overline{\mathbf{J}}$ is a positive semi-definite Hermitian matrix and $\overline{\mathbf{C}}$ is a unitary matrix, then for any unitary matrix $\mathbf{W}$

$$
\|\bar{J}-\mathbf{I}\|=\|\mathbf{J}-\overline{\mathbf{C}}\| \leq\|\mathbf{J}-\mathbf{W}\|
$$

see Fan \& Hoffman (1955). This equation also says that $\overline{\mathbf{J}}$, and all its unitarily similar matrices, is the closest to the unit matrix out of all the unitarily equivalent matrices to $\mathbf{J}$, and hence $\overline{\mathbf{J}}$ is also closest to an ideal polarimeter. This motivates calling $\overline{\mathbf{J}}$ the aligned Jones matrix of $\mathbf{J}$ with respect to $C$, since the unitary transformation of the original Jones matrix $\mathbf{J} \overline{\mathbf{C}}^{\dagger}$ can be seen as aligning the channel basis with the local sky basis. $\check{\mathbf{C}}$ in Eq. (8) is obtained from $\mathbf{C}$ by same unitary transformation as above,

$$
\check{\mathbf{C}}=\mathbf{T}^{\dagger} \overline{\mathbf{C}} \mathbf{T} \text {. }
$$


It is useful to think of $\overline{\mathbf{C}}$ as the empirically determined feed configuration matrix with respect to $\mathcal{S}_{0}$ and $C$.

The leakage matrix D in Eq. (8) has the unique property that it can be understood as the "worst case" of leakage in the aligned polarimeter (the polarimeter given by $\mathbf{J}$ ). Let us define the Jones polarimeter's intrinsic cross-polarization ratio, $\mathrm{IXR}_{\mathrm{J}}$, as the XPIs (or XPDs as they are all equal) in this case, so that

$$
\operatorname{IXR}_{\mathrm{J}} \equiv \frac{1}{d^{2}}
$$

It can be shown that the $\mathrm{IXR}_{\mathrm{J}}$ of the given (generic) Jones matrix $\mathbf{J}$ is the greatest lower bound to both the arithmetic mean of the XPIs over the channels,

$$
\mathrm{IXR}_{\mathrm{J}} \leq \frac{\mathrm{XPI}_{p}+\mathrm{XPI}_{q}}{2}, \quad \text { w.r.t.J, }
$$

and the arithmetic mean of the XPDs over the sky components. The same is also true for the geometric mean: it can be shown that

$$
\mathrm{IXR}_{\mathrm{J}} \leq \sqrt{\mathrm{XPI}_{p} \mathrm{XPI}_{q}}, \quad \text { w.r.t.' }
$$

The XPIs on the right-hand side in these inequalities are with respect to the aligned polarimeters Jones matrix, $\overline{\mathbf{J}}$, while the $\mathrm{IXR}_{\mathrm{J}}$ on the left-hand side is with respect to either $\overline{\mathbf{J}}$ or $\mathbf{J}$, since $\operatorname{IXR}_{\mathrm{J}}(\mathbf{J})=\operatorname{IXR}_{\mathbf{J}}(\overline{\mathbf{J}})$. These inequalities motivate the view of $I_{X R_{J}}$ as the worst-case leakage in a polarimeter.

The property that the IXR $\mathrm{J}_{\mathrm{J}}$ is the minimum XPD can be contrasted with the concept of the maximum XPD, under rotations, put forth in Franco et al. (2003). Note that, for our more general considerations (unitary rather than merely orthogonal transformations), there is no greatest upper bound to an aligned polarimeter's cross-polarization so, for instance, the XPD (or XPI) of a channel (or the average XPD over the channels) can be infinite for some bases. Also, there are no bounds, either lower or upper, for the XPD (or XPI) of $\mathbf{J}$, due to its arbitrariness.

The defining property of the $\mathrm{IXR}_{\mathrm{J}}$ is that it is directly related to the (spectral) condition number $\kappa(\mathbf{J})$ of $\mathbf{J}$ through

$$
\mathrm{IXR}_{\mathrm{J}}=\left(\frac{\kappa(\mathbf{J})-1}{\kappa(\mathbf{J})+1}\right)^{2} .
$$

This follows from the fact that, from equations (5) and (9), we can express the condition number as

$$
\kappa(\mathbf{J})=\frac{1+d}{1-d}
$$

which has the inverse

$$
d=\frac{\kappa(\mathbf{J})-1}{\kappa(\mathbf{J})+1}
$$

and so from Eq. (12) we arrive at Eq. (15). Eq. (15) implies that

$$
1 \leq \mathrm{IXR}_{\mathrm{J}}
$$

since $\kappa \geq 1$. Ideal conditioning $(\kappa=1)$ therefore corresponds to $\mathrm{IXR}_{\mathrm{J}}=0$, or no intrinsic cross-polarization in the polarimeter. Maximum ill-conditioning, $\kappa \rightarrow \infty$, corresponds to $\mathrm{IXR}_{\mathrm{J}}=1$ or maximum intrinsic cross-polarization in the polarimeter. It is interesting to note that equations (16) and (17) are identical to the relations that define the Smith chart transformation.

It is important to understand that, while cross-polarization is sometimes understood as a measure of non-orthogonality of the antenna elements, neither the XPIs, the XPDs nor the IXR generally be understood in this way. For instance, consider two geometrically orthogonal linear dipoles with no mutual leakage, but with imbalanced gains. The Jones matrix is then simply

$$
\mathbf{J}^{(\mathrm{g})}=\left(\begin{array}{cc}
g_{p} & 0 \\
0 & g_{q}
\end{array}\right), \quad g_{p} \neq g_{q} .
$$

For this case, the $\mathrm{IXR}_{\mathrm{J}}$ is therefore

$$
\operatorname{IXR}_{\mathbf{J}}\left(\mathbf{J}^{(\mathrm{g})}\right)=\left(\frac{\left|g_{p}\right|-\left|g_{q}\right|}{\left|g_{p}\right|+\left|g_{q}\right|}\right)^{2}
$$

which is not zero. So even though these channels are orthogonal, the polarimeter intrinsic cross-polarization is non-zero. This is not indicative of a problem in $\mathrm{IXR}_{\mathrm{J}}$. IXR $\mathrm{J}$ is not meant to describe the cross-polarization of a given configuration, but rather it is a measure of the worst-case of leakage out of all configurations of the aligned polarimeter. In the configuration given by Eq. (18), the difference in the gains of the channels leads to a condition number greater than one, and the larger this difference is the more ill-conditioned the Jones matrix is due to the difference in numerical resolution required to measure each channel. This ill-conditioning is equivalent to having a polarimeter with Jones matrix equal to the matrix in Eq. (8) with $d=\left(\left|g_{p}\right|+\left|g_{q}\right|\right) /\left(\left|g_{p}\right|-\left|g_{q}\right|\right)$. The reference frame in this case would be the coordinate system along the $\pm 45^{\circ}$ diagonals to the antennas. Such an $\mathrm{X}$-alignment of antennas is often used by antenna engineers to increase the gain balance between vertical and horizontal components. In passing, we mention that if one is looking for a measure of the antenna non-orthogonality with respect to a fixed channel basis, rather than $\mathrm{IXR}_{\mathrm{J}}$, we suggest using Eq. (27), which is still the polarimeter intrinsic crosspolarization but in the Mueller formalism.

Conversely, there are feed configurations that have non-zero $D$-term leakage but have zero $I_{X R_{\mathrm{J}}}$. To see this, consider a polarimeter with equal gains, $g_{1}=g_{2}=g$ and equal (non-zero) amplitude $D$-leakage terms $d_{p}=d_{q}^{*}=d$. Its Jones matrix is

$$
\mathbf{J}^{(\mathrm{d})}=g\left(\begin{array}{cc}
1 & d \\
-d^{*} & 1
\end{array}\right), \quad|d| \neq 0
$$

but its $\mathrm{IXR}_{\mathrm{J}}$ is zero,

$$
\operatorname{IXR}_{J}\left(\mathbf{J}^{(\mathrm{d})}\right)=0 .
$$

Thus, even though a polarimeter has potentially significant $D$ term leakage, it does not necessarily have any intrinsic crosspolarization.

Summarising this section, we have found a way of expressing the cross-polarization in the Jones matrix of a polarimeter directly and uniquely in terms of the matrix's condition number. This leads us to propose $\mathrm{IXR}_{\mathrm{J}}$ as a single fundamental FoM for the Jones polarimeter that can be used in place of XPI or XPD.

\section{Intrinsic cross-polarization ratio $\left(\mathrm{IXR}_{\mathrm{M}}\right)$ of Stokes polarimeters}

In the previous section we considered the polarimeter in the Jones formalism. In practice many situations require the polarimetric state of a signal to be expressed in terms of Stokes parameters, so we should also consider the previous discussion in terms of the Stokes polarimeter.

While the IXR $\mathrm{IX}_{\mathrm{J}}$, which we introduced in the previous section, relates to the Jones matrix, the analogous quantity for a Stokes 
polarimeter is the Mueller matrix. The Mueller matrix of an arbitrary Jones polarimeter with the Jones matrix $\mathbf{J}$ can be found through the formula

$$
\mathbf{M}=\mathbf{S}^{-1}\left(\mathbf{J} \otimes \mathbf{J}^{*}\right) \mathbf{S}
$$

where $\otimes$ is the Kronecker product and

$$
\mathbf{S} \equiv \frac{1}{2}\left(\begin{array}{cccc}
1 & 1 & 0 & 0 \\
0 & 0 & 1 & \mathrm{i} \\
0 & 0 & -1 & \mathrm{i} \\
1 & -1 & 0 & 0
\end{array}\right)
$$

see, e.g., Hamaker et al. (1996). The Mueller matrix is a real $4 \times 4$ matrix with components $M_{\alpha \beta}$ for $\alpha, \beta=0,1,2,3$.

In the Mueller formalism we take cross-polarization to represent the leakage in the polarimetry between the total power (Stokes $I$ ) and one of the polarized power components (Stokes $Q, U, V)$. This is also sometimes called the instrumental polarization. As with cross-polarization in Jones polarimeters there are two general possibilities: there can be leakage from the total power to a polarized component, or from the polarized component to the total power. We define the former Mueller leakage as

$$
D_{i 0}=\left|\frac{M_{i 0}}{M_{00}}\right|, \quad \text { for } i=1,2,3
$$

and we define the latter Mueller leakage as

$$
D_{0 i}=\left|\frac{M_{01}}{M_{00}}\right|, \quad \text { for } i=1,2,3 .
$$

It can be shown that $D_{i 0} \leq 1$ and $D_{0 i} \leq 1$ for $i=1,2,3$.

As with the Jones polarimeters, the Mueller leakages vary with the choice of local sky basis and channel basis. The similarities with the Jones polarimeter case makes it reasonable to expect the previously introduced concept of intrinsic crosspolarization to be relevant for Stokes polarimeters. We will therefore consider an arbitrary Jones polarimeter which, as we have shown in the previous section (Eq. (8)), can be written $G \mathbf{U D V}^{\dagger}$, where $G$ is total amplitude gain, $\check{\mathbf{D}}$ is the intrinsic feed leakage matrix and $\mathbf{U}$ and $\mathbf{V}$ are two unitary matrices. Its corresponding Mueller matrix is

$$
\mathbf{M}=\frac{G^{2}}{1+d^{2}}\left(\begin{array}{cccc}
1+d^{2} & 2 v_{1} d & 2 v_{2} d & 2 v_{3} d \\
2 u_{1} d & m_{11} & m_{12} & m_{13} \\
2 u_{2} d & m_{21} & m_{22} & m_{23} \\
2 u_{3} d & m_{31} & m_{32} & m_{33}
\end{array}\right),
$$

where $u_{i}, v_{i} \in \mathbb{R}$ for $i=1,2,3$, and $m_{i j}$, for $i, j=1,2,3$, are functions of $u_{i}, v_{i}$, and $d$. The parameters $u_{1}, u_{2}, u_{3}$ and $v_{1}, v_{2}, v_{3}$ depend on $\mathbf{U}$ and $\mathbf{V}$ as follows:

$$
\begin{aligned}
& u_{1}=2 \mathfrak{R}\left(U_{11} U_{12}\right) \\
& u_{2}=-\mathfrak{R}\left(U_{11}^{2}-U_{12}^{2}\right) \\
& u_{3}=\mathfrak{J}\left(U_{11}^{2}-U_{12}^{2}\right)
\end{aligned}
$$

and

$$
\begin{aligned}
& v_{1}=2 \mathfrak{R}\left(V_{11} V_{12}\right) \\
& v_{2}=-\mathfrak{R}\left(V_{11}^{2}-V_{12}^{2}\right) \\
& v_{3}=\mathfrak{T}\left(V_{11}^{2}-V_{12}^{2}\right),
\end{aligned}
$$

and they fulfill

$$
u_{1}^{2}+u_{2}^{2}+u_{3}^{2}=v_{1}^{2}+v_{2}^{2}+v_{3}^{2}=1 .
$$
for $\mathbf{M}$

From Eq. (21) one can easily read off the Mueller leakages

$$
\begin{aligned}
& D_{i 0}=\frac{2 u_{i} d}{1+d^{2}} \\
& D_{0 i}=\frac{2 v_{i} d}{1+d^{2}}
\end{aligned}
$$

which clearly depend on $u_{i}$ and $v_{i}$ respectively, but their root sum square

$$
\sqrt{D_{10}^{2}+D_{20}^{2}+D_{30}^{2}}=\sqrt{D_{01}^{2}+D_{02}^{2}+D_{03}^{2}}=\frac{2 d}{1+d^{2}} \equiv D
$$

does not depend on either $u_{i}$ nor $v_{i}$. We therefore introduce $D$ as the Mueller intrinsic leakage term, echoing the results of the previous section.

If we consider cross-polarization in the Mueller formalism to be the reciprocal of the Mueller leakages, then we can generate an IXR in the Mueller formalism as

$$
\mathrm{IXR}_{\mathrm{M}}=\frac{1}{D},
$$

which we will call the Mueller polarimeter intrinsic crosspolarization ratio $\left(\mathrm{IXR}_{\mathrm{M}}\right)$. As with $\mathrm{IXR}_{\mathrm{J}}, \mathrm{IXR}_{\mathrm{M}}$ is also a lower bound on the Mueller cross-polarizations,

$$
\mathrm{IXR}_{\mathrm{M}} \leq \frac{1}{D_{0 i}}, \quad \text { for } i=1,2,3
$$

and

$$
\mathrm{IXR}_{\mathrm{M}} \leq \frac{1}{D_{i 0}}, \quad \text { for } i=1,2,3 .
$$

Note however, that this is valid only for the class of Mueller polarimeters that can be constructed according to Eq. (20), that is, non-depolarizing polarimeters.

The similarity between IXR $\mathrm{IJ}_{\mathrm{J}}$ and $\mathrm{IXR}_{\mathrm{M}}$ is not confined to similarities in leakage bounds, it extends also to the relation with their respective matrix condition numbers. In the case of the Mueller matrix, its condition is

$$
\kappa(\mathbf{M})=\kappa\left(\mathbf{J} \otimes \mathbf{J}^{*}\right)=\frac{\sigma_{\mathrm{M}}^{2}}{\sigma_{\mathrm{m}}^{2}}=\kappa^{2}(\mathbf{J}) .
$$

So while $\mathrm{IXR}_{\mathrm{J}}$ was related to the condition number $\kappa(\mathrm{J})$ through a Smith chart-type transformation, (see Eq. (17)), we find that $\mathrm{IXR}_{\mathrm{M}}$ has exactly the same relation to $\kappa(\mathbf{M})$, that is,

$$
D=\frac{\kappa(\mathbf{M})-1}{\kappa(\mathbf{M})+1}=\frac{\kappa^{2}(\mathbf{J})-1}{\kappa^{2}(\mathbf{J})+1} .
$$

The inverse of this transformation is

$$
\kappa(\mathbf{M})=\kappa^{2}(\mathbf{J})=\frac{1+D}{1-D}=\left(\frac{1+d}{1-d}\right)^{2} .
$$

This clearly shows that both $\mathrm{IXR}_{\mathrm{M}}$ and $\mathrm{IXR}_{\mathrm{J}}$ are directly related to the condition number of both the Mueller matrix and the Jones matrix. Fig. 2 shows the strictly monotonic relationships between IXR $, I_{\mathrm{J}}, \mathrm{R}_{\mathrm{M}}$ and the condition number of the Jones (or Mueller) matrix.

The $\mathrm{IXR}_{\mathrm{M}}$ is related to diattenuation as defined in Lu \& Chipman (1994). Indeed the two parameters are equal when the Jones matrix is homogeneous since in this case the singular values of the Jones matrix are equal to its eigenvalues. 


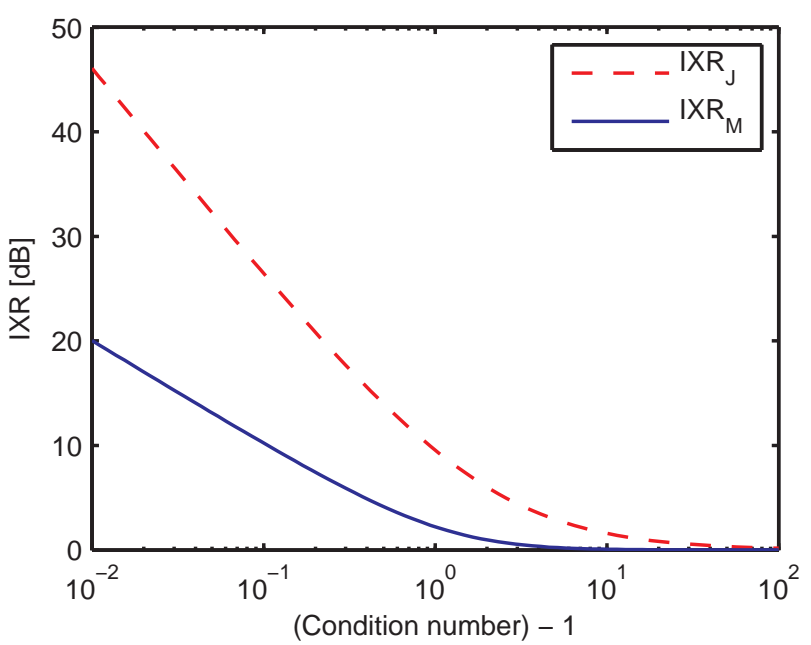

Figure 2. Plots of $I X R_{J}$ and $I X R_{M}$ as functions of the condition number of the Jones matrix minus one.

In the above we have looked at polarimeters that are characterised by their Jones matrices (non-depolarizing polarimeters), but there are also polarimeters that only have a Mueller matrix $\mathbf{M}$. For such polarimeters $\mathrm{IXR}_{\mathrm{J}}$ is of course not defined, but the $\mathrm{IXR}_{\mathrm{M}}$ can still be defined as

$$
\mathrm{IXR}_{\mathrm{M}} \equiv \frac{\kappa(\mathbf{M})+1}{\kappa(\mathbf{M})-1}
$$

Having shown how leakage in the Mueller formalism can be expressed in terms of Jones matrix leakage we can now show the converse, expressing leakage in the Jones formalism in terms of leakage in the Mueller matrix. We do this in following way: imagine that we wanted an invariant expression for the leakage between the $p$ and $q$ channels of a Jones polarimeter with the Jones matrix $\mathbf{J}$. Let us take the first row in $\mathbf{J}$ to be the vector $\mathbf{J}_{p} \equiv\left(\begin{array}{ll}J_{p 1} & J_{p 2}\end{array}\right)$ and the second row to be the vector $\mathbf{J}_{q}=\left(\begin{array}{ll}\mathrm{J}_{q 1} & \mathrm{~J}_{q 2}\end{array}\right)$, and let us call them the channel vectors of the polarimeter. The channel vectors of the polarimeter are similar to effective antenna length vectors, but include the effects of polarimeter leakage, etc. In analogy with the polarization efficiency parameter between a transmitting antenna and a receiving antenna, which is defined as the normalised inner product between their effective antenna length vectors (Stutzman 1993), we introduce the normalised inner product of the polarimeter channel vectors as a measure of the non-orthogonality between channel vectors. This parameter can be understood as a (sky invariant) measure of the leakage between the $p$ and $q$ channels, and it is easy to show that this inner product fulfills

$$
\frac{\left|\mathbf{J}_{p} \cdot \mathbf{J}_{q}^{*}\right|}{\left|\mathbf{J}_{p}\right|\left|\mathbf{J}_{q}\right|} \leq D
$$

where $D$ is the Mueller polarimeter intrinsic leakage for Mueller matrix corresponding to $\mathbf{J}$. This means that the Mueller polarimeter intrinsic leakage sets the upper bound (or worst-case scenario) for the leakage between the channel vectors of the polarimeter.

\section{Interferometric polarimeters}

Having developed IXRs for Jones- and Mueller-based radio polarimeters our final concern is with interferometric polarimeters.
There are many different types of radio interferometers, but we will only examine interferometers comprising a combination of at least two Jones polarimeters. Although we have already considered Jones polarimeters, the models for the interferometer response are sufficiently different to warrant special attention.

The most fundamental model of a radio interferometer is expressed in terms of coherence matrices. For our proposes we only need to consider the simplest case: a single partially polarized point source in the direction $\mathbf{s}$ with a brightness coherence matrix $\mathbf{B}$ measured by a two-element interferometer. If elements $A$ and $B$ have (approximately) known Jones matrices, $\mathbf{J}_{\mathrm{A}}(\mathbf{s})$ and $\mathbf{J}_{\mathrm{B}}(\mathbf{s})$, the interferometer output is the visibility coherence matrix $\mathbf{V}_{\mathrm{AB}}$. The response of the interferometer is called the (paraxial) measurement equation of radio interferometry, and in this case is simply

$$
\mathbf{V}_{\mathrm{AB}}=\mathbf{J}_{\mathrm{A}}(\mathbf{s}) \mathbf{B}(\mathbf{s}) \mathbf{J}_{\mathrm{B}}^{\dagger}(\mathbf{s}) \exp \left(+\mathrm{i} \mathbf{D}_{\mathrm{AB}} \cdot \mathbf{s}\right)
$$

where $\mathbf{D}_{\mathrm{AB}}$ is the baseline vector between the positions of $\mathrm{A}$ and B. If $\mathbf{J}_{\mathrm{A}}^{-1}$ and $\left(\mathbf{J}_{\mathrm{B}}^{\dagger}\right)^{-1}$ exist, the solution to this measurement equation is

$$
\mathbf{B}(\mathbf{s})=\mathbf{J}_{\mathrm{A}}^{-1} \mathbf{V}_{\mathrm{AB}}\left(\mathbf{J}_{\mathrm{B}}^{\dagger}\right)^{-1} \exp \left(-\mathrm{i} \mathbf{D}_{\mathrm{AB}} \cdot \mathbf{s}\right) .
$$

The important point here is that even if $\mathbf{J}_{\mathrm{A}}$ and $\mathbf{J}_{\mathrm{B}}$ are very good estimates, the estimate of the brightness matrix, Eq. (29), can still be poor if $\mathbf{J}_{\mathrm{A}}$ or $\mathbf{J}_{\mathrm{B}}$ are ill-conditioned. This is because in practice there is noise in all the measurements, and illconditioning in the Jones matrices acts as noise amplification. In the worst-case scenario, one or both of the Jones matrices are singular (with infinite condition number) so Eq. (29) is not valid and a full solution is not possible. Thus Eq. (29) suggests that the condition number of the Jones polarimeter elements that make up the interferometer relate to the accuracy of the brightness estimates. In other words, the IXR $\mathrm{I}_{\mathrm{J}}$ of the individual Jones matrices of an interferometer can be used to assess the total polarimetry of the interferometer.

A more holistic assessment of a Jones interferometer can be found if we express the measurement equation in terms of Stokes vectors. This involves converting the polarimetric response from a Jones formalism to a Mueller formalism. The Mueller matrix that corresponds to the interferometer in Eq. (28) is

$$
\mathbf{M}_{\mathrm{AB}}=\mathbf{S}^{-1}\left(\mathbf{J}_{\mathrm{A}} \otimes \mathbf{J}_{\mathrm{B}}^{*}\right) \mathbf{S}
$$

where we have put the phase reference centre $\mathbf{s}=0$ along source direction. Characteristically, this interferometric Mueller matrix $\left(\mathbf{M}_{\mathrm{AB}}\right)$ is complex rather than real, mapping brightness Stokes vectors to visibility Stokes vectors.

For an interferometer with $N$ Jones polarimeter elements, the total interferometric Mueller matrix is the sum of all contributions from the interferometer arms, Eq. (30), so

$$
\mathbf{M}_{\text {Int }}=\mathbf{S}^{-1}\left(\sum_{\substack{i=1 \\ j}}^{N} \sum_{\substack{j=1 \\ j \neq i}}^{N} \mathbf{J}_{i} \otimes \mathbf{J}_{j}^{*}\right) \mathbf{S}
$$

for the direction along the phase reference centre, which we have set to $\mathbf{s}=0$.

Unlike the ordinary (real) Mueller matrix, the interferometer Mueller matrix involves visibilities and is complex, but the definition of IXR $\mathrm{I}_{\mathrm{M}}$ in Eq. (26) can still be used, and its interpretation is analogous to our earlier discussion.

One should note that for imaging interferometric polarimeters, errors in the polarimetry do not only affect the measurement 
of the state-of-polarization of a source, but also the measurement of its direction, see, e.g., Bhatnagar \& Nityananda (2001). Thus IXR is also a measure of the pointing accuracy in interferometric imaging.

\section{Examples}

In this section we compute the IXR and $\mathrm{IXR}_{\mathrm{M}}$ parameters for a selection of radio polarimeters, based mostly on data in the public domain. The results we present should in no sense be seen as an authoritative assessment of the performance of the respective polarimeters, but rather as illustrations of how the polarimetric parameters can be used and the sort of values these parameters may typically attain. For this reason we refrain from passing judgments on the individual instruments and only mention that in general (barring all caveats) the higher the values of IXR, the better the polarimeter, according to the assertions made in this paper.

\subsection{Parameterisation of Jones matrices for the Parkes Telescope}

van Straten (2004) determines the Jones matrix for the Parkes telescope over a range of frequencies. Based on Jones matrices similar to these, we compute the $\mathrm{IXR}_{\mathrm{J}}$ for the Parkes polarimeter.

van Straten (2004) employs two parametrization of the Jones matrix: one is what he calls the "phenomenological parameterisation" based on Britton (2000), and the other is what he calls the "algebraic parameterisation" based on Hamaker et al. (1996). In both cases the Jones matrices are obtained by fitting observed Stokes pulsar profiles to templates of pulsar profiles.

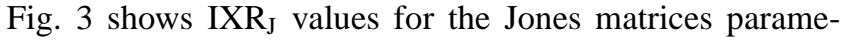
terised according to these two parameterisations over a range of frequencies. We see that Parkes performs 'better' in the phenomenological parameterisation than in the algebraic parameterisation over the entire frequency range. Since the polarimeter is the same in both parameterisations the differences are most likely due to differences in the numerical accuracies of the parameterisations at the template matching stage. Although it is not entirely obvious which of the two parameterisation is more correct, it is intuitive to think that the lower the numerical accuracy in the computation of a Jones matrix, the more ill-conditioned that Jones matrix would be, and hence it is natural to expect the phenomenological parameterisation to be the more correct of the two.

This example suggests that the $\mathrm{IXR}_{\mathrm{J}}$ could be used not only to assess polarimeters themselves, but also to assess a calibration of the polarimeter.

\subsection{The Effelsberg Telescope}

Xilouris (1991) presented a Mueller matrix for the Effelsberg telescope. Based on this matrix we find that the Effelsberg telescope, when seen as a Stokes polarimeter, has $\mathrm{IXR}_{\mathrm{M}}=9.7 \mathrm{~dB}$. This corresponds to a polarimeter intrinsic Mueller leakage of $10.7 \%$. However, the root mean square of its instrumental polarization, $\sqrt{\left|M_{10}\right|^{2}+\left|M_{20}\right|^{2}+\left|M_{30}\right|^{2}} /\left|M_{00}\right|$ where $M_{i j}$ are the components of the Mueller matrix, is $11.0 \%$, while $\sqrt{\left|M_{01}\right|^{2}+\left|M_{02}\right|^{2}+\left|M_{03}\right|^{2}} /\left|M_{00}\right|$ is $10.6 \%$. Thus in this case, $\mathrm{IXR}_{\mathrm{M}}$ is the worst-case leakage for $D_{0 i}$, but not for $D_{i 0}$. This does not violate the result of the previous section (Eqs. (25)): this Mueller matrix exhibits depolarization,

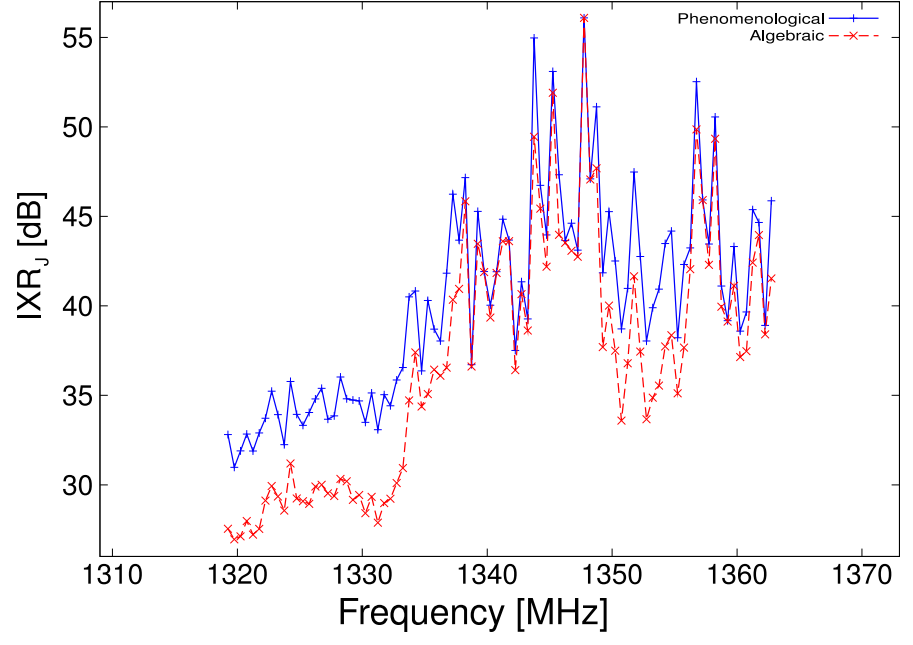

Figure 3. The IXR $\mathrm{I}_{\mathrm{J}}$ of the Parkes telescope for various frequencies based on two different parameterisations of the Jones matrix. The two Jones matrix parameterisation are the phenomenological (full line) and the algebraic (dashed line). The two show a clear difference, with the phenomenological parameterisation having a better IXR $\mathrm{J}_{\mathrm{J}}$ in this case.

as $\sqrt{\left|M_{01}\right|^{2}+\left|M_{02}\right|^{2}+\left|M_{03}\right|^{2}} \neq \sqrt{\left|M_{10}\right|^{2}+\left|M_{20}\right|^{2}+\left|M_{30}\right|^{2}}$ as required by Eq. (21).

\subsection{Off-axis aberrations in a short dipole interferometer}

In this example we compute analytically the $\mathrm{IXR}_{\mathrm{J}}$ for crossed dipoles as function of incidence direction. The crossed dipoles are fixed to the ground and set up so their symmetry axis is vertical, pointing to zenith. The model is an approximation of several future radio telescopes, including LOFAR and SKA, at low frequencies.

Carozzi \& Woan (2009), derived the Jones matrix in this case to be

$$
\mathbf{J}(l, m)=\frac{1}{\sqrt{1-m^{2}}}\left(\begin{array}{cc}
\sqrt{1-l^{2}-m^{2}} & -l m \\
0 & 1-m^{2}
\end{array}\right)
$$

where $l, m$ are the direction cosines of the source. We can determine the condition of this Jones matrix by computing the eigenvalues of $\mathbf{J} \mathbf{J}^{\dagger}$ and taking the square root of their ratio. The result is

$$
\kappa(\mathbf{J})=\frac{1}{\sqrt{1-l^{2}-m^{2}}}
$$

so the $\mathrm{IXR}_{\mathrm{J}}$ is

$$
\mathrm{IXR}_{\mathrm{J}}=\left(\frac{1+\sqrt{1-l^{2}-m^{2}}}{1-\sqrt{1-l^{2}-m^{2}}}\right)^{2} .
$$

Fig. 4 shows the dependence of $\mathrm{IXR}_{\mathrm{J}}$ on the zenith angle, equal to $\arcsin \left(\sqrt{l^{2}+m^{2}}\right)$ ), according to Eq. (33). If one were to design an array in which the scientific objectives required measurements better than some prescribed cross-polarization, then this curve shows the limit of the maximum possible field-ofview (FoV) that could be attained. Vice versa, if the design had a prescribed FoV, then this curve gives the best possible crosspolarization ratio at the edges of the FoV. For a FoV of, say, 250 square degrees, the edge of the FoV will have an IXR ${ }_{J}$ of $45 \mathrm{~dB}$. 


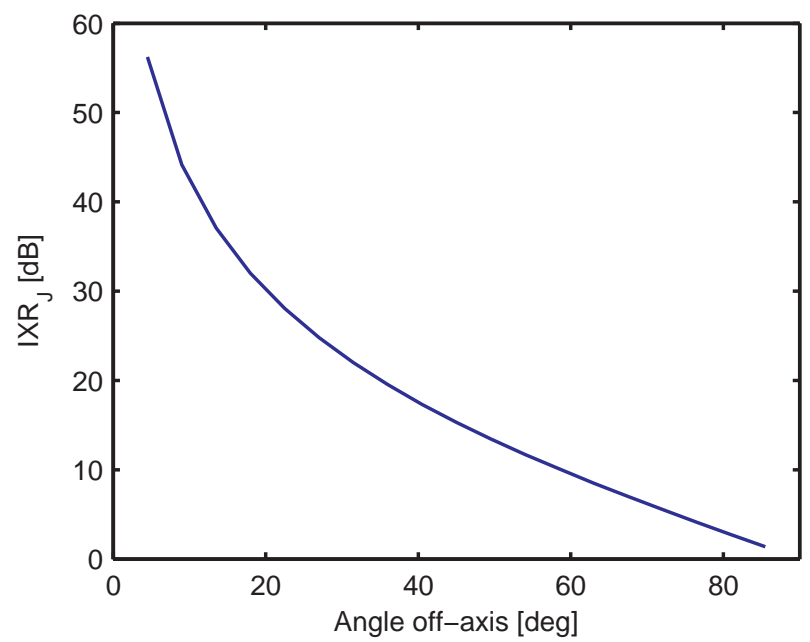

Figure 4. $\mathrm{IXR}_{\mathrm{J}}$ as a function of the zenith angle for short dipole interferometers.

It is also interesting to consider the $\mathrm{IXR}_{\mathrm{M}}$ in this case since Carozzi \& Woan (2009) used a Mueller matrix based on the Jones matrix in Eq. (32) to show that short dipole interferometers are polarimetrically aberrated. With the $\mathrm{IXR}_{\mathrm{M}}$ introduced in the present paper, we can now assess more concisely the polarimetry in this case. The result is

$$
\mathrm{IXR}_{\mathrm{M}}=\frac{2-l^{2}-m^{2}}{l^{2}+m^{2}}
$$

This agrees with the ratio of the measured polarized power to the measured unpolarized power for unpolarized radiation according to the Mueller matrix presented in Carozzi \& Woan (2009).

\subsection{The WSRT Interferometer}

The Westerbork synthesis radio telescope (WSRT) is an EastWest interferometer consisting of fourteen, 25-m parabolic dishes with dual-polarized feeds.

We analysed estimates of the Jones matrices for each of the 14 dual linearly-polarized feeds over a band of frequencies, based on a dataset similar to that used in Brentiens (2008). For one of the frequency channels, centred on $346.0 \mathrm{MHz}$, we computed the IXR $\mathrm{IX}_{\mathrm{M}}$ of each the individual polarimeters and the $\mathrm{IXR}_{\mathrm{M}}$ of all 14 taken together as an interferometer.

The IXR $\mathrm{IM}_{\mathrm{M}}$ value for each of the 14 dishes is plotted in Fig.5, along with the $\mathrm{IXR}_{\mathrm{M}}$ of the interferometer based on Eq. (31). We see that the interferometric $I_{X R_{M}}$ is, here, approximately an average of the individual $\mathrm{IXR}_{\mathrm{M}}$ values. Thus, in this case, the whole is not much better than its parts. From Eq. (31), this may suggest that the Jones matrices for the individual telescopes can be modelled as having small, random deviations from a nonideal mean Jones matrix.

Naturally these results are specific to the feed used in this case, and different results are expected for other WSRT feeds.

\section{Discussion}

We have introduced the polarimeter intrinsic cross-polarization ratio parameters $I_{X R_{J}}$ and $I_{X R_{M}}$, which can be considered as two versions of one concept: the polarimeter intrinsic crosspolarization ratio, (IXR). They differ mainly in whether the

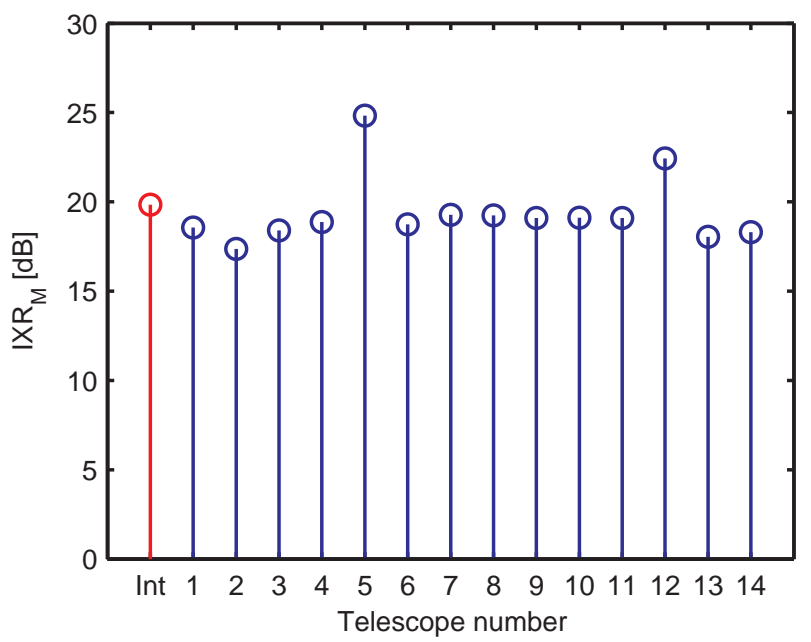

Figure 5. The IXR $\mathrm{IX}_{\mathrm{M}}$ values of the WSRT at frequency $346 \mathrm{MHz}$. The $\mathrm{IXR}_{\mathrm{M}}$ of the individual polarimeters are in given in $\mathrm{dB}$ as a function of the telescope number. The telescope number marked "Int" represents all of the 14 telescopes taken together as an interferometer.

cross-polarization refers to a Jones polarimeter or a Stokes polarimeter, respectively. We now discuss the proposal of using IXR as a FoM of a polarimeter rather than a parameter such as XPI in radio astronomical polarimetry. The utility of the IXR parameter is in its dual propose use in assessing both calibrated and uncalibrated polarimeters.

For uncalibrated polarimetry, XPI is essentially a signal-tonoise (SNR) parameter. It gives the ratio of desired power to undesired power when there is an equal amount of power in both of the Jones vector components. In such a context, the IXR parameter serves as a FoM in that it represents the worst-case scenario for that particular polarimeter. On the other hand, XPI depends on the choice of coordinate system. In any case it is important to note that the XPI is not, in general, a measure of the error in the fully calibrated polarimetry.

The main justification for using IXR as a polarimeter FoM is however its direct relation to the condition number of the polarimetric response matrix, making it a good indicator of the relative errors in the final, calibrated, polarimetric measurement. This property of the polarimeter is completely independent of the particular choice for the sky and channels bases, as one would expect from an intrinsic FoM characterising the physical instrument. For a full calibration, the relative error in the estimated Jones vector, $\|\Delta \mathbf{e}\| /\|\mathbf{e}\|$, can be shown to be (Lancaster \& Tismenetsky 1985)

$$
\frac{\|\Delta \mathbf{e}\|}{\|\mathbf{e}\|} \leq \frac{\kappa}{1-\kappa\|\Delta \mathbf{J}\| /\|\mathbf{J}\|}\left(\frac{\|\Delta \mathbf{J}\|}{\|\mathbf{J}\|}+\frac{\|\Delta \mathbf{f}\|}{\|\mathbf{f}\|}\right),
$$

where $\|\Delta \mathbf{J}\| /\|\mathbf{J}\|$ is the total relative error in the calibration of the polarimeter's Jones matrix $\mathbf{J},\|\Delta \mathbf{f}\|^{2} /\|\mathbf{f}\|^{2}$ is the reciprocal of the polarimeter's signal-to-noise ratio (SNR) and $\kappa$ is the condition number of $\mathbf{J}$. Most practical radio polarimeters will have $\mathrm{IXR}_{\mathrm{J}} \gg 1$ and will be well calibrated, so $\|\Delta \mathbf{J}\| /\|\mathbf{J}\| \ll d$ (where $d=1 / \sqrt{\mathrm{IXR}_{\mathrm{J}}}$ ). Under these conditions we can rewrite the inequality (34) as

$$
\frac{\|\Delta \mathbf{e}\|}{\|\mathbf{e}\|} \lesssim\left(1+\frac{2}{\sqrt{\mathrm{IXR}_{\mathbf{J}}}}+\ldots\right)\left(\frac{\|\Delta \mathbf{J}\|}{\|\mathbf{J}\|}+\frac{\|\Delta \mathbf{f}\|}{\|\mathbf{f}\|}\right) .
$$


It is clear from Eq. (35) that, for fixed $\|\Delta \mathbf{J}\| /\|\mathbf{J}\|$ and $\|\Delta \mathbf{f}\| /\|\mathbf{f}\|$, the $I_{X R}$ is directly related to the total relative error of the polarimeter's measurement of the Jones vector. The best (extreme) case is when $\mathrm{IXR}_{\mathrm{J}} \rightarrow \infty$, then the total relative error is just the sum of the total relative errors in the Jones measurements and the Jones matrix measurements. In general, the smaller the $\mathrm{IXR}_{\mathrm{J}}$, the larger the error.

Although we have focused mainly on the IXR as a FoM for the polarimeter instrument itself, the IXR can also be used to assess aspects related to more general polarimetry. We saw in Section 7.1 that the IXR effectively assessed the calibration of the polarimeter. Another extended use of the IXR is in the assessment of the complete measurement equation of a polarimeter: all the phenomena that affect the polarimetry from the source to the output signal can be expressed in terms of matrix factors that together make up a total effective Jones matrix for the polarimetry. The IXR can be determined for this total, effective, Jones matrix and can be understood as an assessment of the polarimetry associated with that particular measurement equation. The lower the IXR of the effective Jones matrix of a measurement equation, the more difficult it will be to obtain high dynamic range for the full polarimetry. In the extreme, but practically feasible, case that the IXR is zero, full polarimetry is unobtainable as the effective Jones matrix is singular.

The discussion above has been about the IXR as a function of the source direction. Often, however, it is desirable to have a FoM which is some aggregate over all directions. We will not pursue this line of inquiry further here except to mention that there are several possibilities for the overall polarimeter FoM based on the direction dependent IXR, including the maximum IXR over all directions, the IXR along the boresight, or simply the IXR weighted with the gain, averaged over all directions.

To be useful and practical a FoM should of course be measurable, preferably with minimal effort. As a final point in this section we mention that the IXR is well-suited also in the respect. This is because it is not necessary to measure the full Jones, or Mueller, matrix to determine a polarimeter's IXR. It is sufficient to measure the polarimeter's response to unpolarized radiation only, which is much easier than a full calibration which ordinarily requires at least three different polarized fiducial measurements. The reason for this is that the IXR is based just on the invariants of the Jones or Mueller matrices and not on the full Jones or Mueller matrices themselves. For the XPI and $\mathrm{XPD}$, on the other hand, the full Jones matrix is required making them generally harder to compute.

\section{Conclusions}

We find that the standard cross-polarization ratio parameters are extrinsic properties of a polarimeter, that is, they depend on an arbitrary choice of coordinate system. We argue that this makes it difficult to interpret these standard cross-polarizations as a genuine, intrinsic, leakage in a polarimeter. Furthermore, they are not meaningful after full calibrations and for these reasons we find that the standard cross-polarization ratios should not be treated as a polarimeter figure of merit. However, we suggest that the worst-case cross-polarization of the (aligned) polarimeter is unique and invariant, and so could serve as the intrinsic polarimeter leakage. We present a parameter, which we call the intrinsic polarimeter cross-polarization ratio (IXR) that satisfies this worst case. We show how the parameter can be applied to the various basic types of radio polarimeters and how it ultimately determines the total relative error in a polarimeter. This last property qualifies IXR as a fundamental figure of merit for radio polarimeters. We also find that IXR can be used not only to assess the polarimeters themselves, but also to assess a particular calibration of a polarimeter.

Acknowledgements. We thank Michiel Brentjens for providing Jones matrices of the WSRT. We also thank Willem van Straten for providing the Jones matrices of the Parkes telescope. This work is supported by the European Community Framework Programme 6, Square Kilometre Array Design Studies (SKADS), contract no 011938, and the Science and Technology Facilities Council (STFC), UK.

\section{References}

Bhatnagar, S. \& Nityananda, R. 2001, A\&A, 375, 344

Brentjens, M. A. 2008, A\&A, 489, 69

Britton, M. C. 2000, Ap. J., 532, 1240

Carozzi, T. D. \& Woan, G. 2009, MNRAS

Fan, K. \& Hoffman, A. J. 1955, Proceedings of the American Mathematical Society, 6, 111

Franco, G., Fosalba, P., \& Tauber, J. A. 2003, A\&A, 405, 349

Hamaker, J. P., Bregman, J. D., \& Sault, R. J. 1996, Astron. Astrophys. Suppl. Ser., 117,137

IEEE. 1998, IEEE Std 211-1997

Lancaster, P. \& Tismenetsky, M. 1985, The Theory of Matrices, 2nd edn. (Academic Press)

Leppänen, K., Massi, M., Rioja, M., \& Sanghera, H. 1997, Vistas in Astronomy, 41,291 , proceedings of the 3rd EVN/JIVE VLBI Symposium

Lu, S.-Y. \& Chipman, R. A. 1994, J. Opt. Soc. Am. A, 11, 766

Ludwig, A. 1973, Antennas and Propagation, IEEE Transactions on, 21, 116

Massi, M., Rioja, M., Gabuzda, D., et al. 1997, Vistas in Astronomy, 41, 287 , proceedings of the 3rd EVN/JIVE VLBI Symposium

Reid, R. I., Gray, A. D., Landecker, T. L., \& Willis, A. G. 2008, Radio Science, 43, 2008

Sabatke, D. S., Locke, A. M., Descour, M. R., et al. 2000, in Polarization Analysis, Measurement, and Remote Sensing III, ed. D. B. Chenault, M. J. Duggin, W. G. Egan, \& D. H. Goldstein, Vol. 4133 (SPIE), 75-81

Sault, R. J., Hamaker, J. P., \& Bregman, J. D. 1996, Astron. Astrophys. Suppl. Ser., 117,149

Stutzman, W. L. 1993, Polarization in electromagnetic systems (Artech house, Inc.)

Thiel, M. A. F. 1976, J. Opt. Soc. Am., 66, 65

van Straten, W. 2004, Astrophys. J. Suppl. Ser., 152, 129

Xilouris, K. M. 1991, A\&A, 248, 323 